\title{
A Suplementação de Carboidrato Maximiza o Desempenho de Tenistas?
}

\section{Does Carbohydrate Supplementation Maximize Performance of Tennis Players?}

Rodrigo Vitasovic Gomes' Marcelo Saldanha Aoki ${ }^{1,2}$

1. Escola de Educação Física e Esporte da Universidade de São Paulo, São Paulo, Brasil.

2. Escola de Artes, Ciências e Humanidades da Universidade de São Paulo, São Paulo, Brasil.

\section{Endereço para correspondência:} Marcelo Saldanha Aoki.

Grupo de Pesquisa em Adaptações Biológicas ao Exercício Físico. Escola de Artes, Ciências e Humanidades.

Universidade de São Paulo. Av. Arlindo Bettio, 1.000. 03828-000 - São Paulo, SP, Brasil. E-mail: saldanha.caf@usp.br

\begin{abstract}
RESUMO
O tênis é um esporte complexo, influenciado por muitas variáveis, tais como o tipo de quadra (lenta ou rápida), o tipo de bola e o padrão tático do jogador (ofensivo ou defensivo). Esse esporte é considerado uma atividade intermitente de longa duração que, provavelmente, recruta diferentes tipos de substratos energéticos. Portanto, devido às características do tênis, é plausível admitir que o carboidrato seja um importante combustível para essa atividade. O efeito ergogênico do carboidrato já foi comprovado no exercício de endurance. Entretanto, no tênis, poucos estudos investigaram o papel desse nutriente sobre o desempenho. O objetivo do presente artigo é apresentar e discutir os estudos disponíveis sobre os efeitos da suplementação de carboidrato no desempenho de tenistas. A literatura atual apresenta escasso número de estudos, com o agravante dos mesmos apresentarem resultados controversos. Portanto, os poucos estudos não permitem que a pergunta levantada no título do artigo seja respondida de maneira satisfatória. A controvérsia observada nos estudos é, provavelmente, consequência de modelos experimentais diferentes, tais como: a duração do treino/jogo/teste, os parâmetros utilizados para medir desempenho, o conteúdo inicial dos estoques de glicogênio e a análise/controle da dieta antes do experimento. Estudos adicionais, em condições reais de jogo, precisam ser conduzidos, a fim de avaliar o real efeito da suplementação de carboidrato sobre o desempenho no tênis.
\end{abstract}

Palavras-chave: nutrição, tênis, glicemia, glicogênio.

\begin{abstract}
Tennis is a complex sport influenced by many variables, such as the type of court (slow or fast), the ball used and the tactical pattern of the player (offensive or defensive). It is considered a long lasting intermittent activity that probably recruits different energy substrates. Thus, due to its specific characteristics, it is reasonable to expect that carbohydrate might be an important energy substrate for this sport. The ergogenic role of carbohydrate for endurance sports is well known. However, only few studies investigated the role of this nutrient during tennis practice. The aim of the present work is to discuss the data regarding the effect of carbohydrate supplementation on tennis performance. There are only few studies available with controversial results. Therefore, it is not currently possible to draw a final conclusion on this topic. Probably, the controversy is related to different experimental models. Further studies should be carried out in order to assess the real effect of carbohydrate supplementation on tennis performance.
\end{abstract}

Keywords: nutrition, tennis, glycaemia, glycogen.

\section{INTRODUÇÃO}

O objetivo deste trabalho é apresentar e discutir os estudos disponíveis sobre os efeitos da suplementação de carboidrato $(\mathrm{CHO})$ no desempenho de tenistas. Para responder ao questionamento levantado no título do artigo foi realizada uma busca no banco de artigos do PubMed (www.pubmed.gov), utilizando os descritores em inglês: tennis (tênis), carbohydrate (carboidrato) e supplementation (suplementação). Apesar da grande popularidade do esporte, os estudos sobre estratégias nutricionais no tênis são escassos. Nesta revisão da literatura serão, inicialmente, abordadas as demandas fisiológicas do tênis. Logo após, será apresentado um breve histórico sobre a suplementação de $\mathrm{CHO}$ no exercício de endurance, seguido pelo tópico referente aos estudos disponíveis sobre suplementação de CHO no tênis.

\section{Caracterização do tênis}

O tênis é um esporte que atrai milhões de jogadores e fãs no mundo inteiro. O calendário de competições acontece ao longo do ano, sob supervisão e regras da Federação Internacional de Tênis (ITF). Existem vários tipos de competições, os "Grand Slams", os "Master Series" e as competições de transferência do nível amador para o profissional, como os "Futures" e os "Challengers". Estima-se que sejam realizadas aproximadamente de 60 (WTA) a 80 (ATP) torneios por ano, tanto para mulheres quanto para homens ${ }^{(1)}$.

Esse esporte apresenta caráter intermitente, intercalando movimentos explosivos de alta intensidade e curta duração com períodos de recuperação(1). As ações específicas do tênis são caracterizadas por saídas rápidas com paradas bruscas, mudanças de direção e golpes 
realizados com a raquete (ex:: smash, forehand, backhand, saque, etc.).(1). Já os intervalos têm duração máxima de 20 segundos entre os pontos, 90 segundos entre as trocas de lado nos games ímpares e 120 segundos entre os sets ${ }^{(2)}$. Vale ressaltar que, desde 2004, os períodos de recuperação são controlados pelas regras da ITF. Além disso, o tênis apresenta algumas características singulares: esse é o único esporte praticado em diferentes pisos, com diferentes bolas e com duração bastante variada (melhor de três sets ou melhor de cinco sets) $)^{(1)}$.

Essas particularidades do tênis tornam a quantificação do nível de esforço de uma partida bastante complexa, exigindo a análise de diversos critérios (tempo total de jogo, tempo real de jogo, tempo de troca de bolas, distância percorrida, comportamento da frequência cardíaca, concentração de lactato, percepção subjetiva do esforço, etc.). Conforme já mencionado, a maioria das ações no tênis é realizada em alta intensidade e curtíssima duração (4-10 segundos) $)^{(3,4)}$, no entanto, o tempo total de uma partida de tênis varia de uma a cinco horas. Apesar da longa duração total, o tempo real de jogo é de aproximadamente $20-30 \%$ do tempo total em quadras lentas (saibro) e 10-15\% em superfícies mais rápidas (asfalto, carpete ou grama) ${ }^{(3)}$. Nesse período (tempo real), o jogador percorre em média três metros por golpe, totalizando oito a 12 metros por ponto(3,4,5). Estima-se que aproximadamente $80 \%$ dos golpes sejam executados a 2,5 metros da posição inicial, 10\% a 2,5 a 4,5 metros e, apenas, 5\% acima de 4,5 metros ${ }^{(4,6)}$. Outro parâmetro a ser considerado é a duração média de cada ponto. O tempo de troca de bolas é ligeiramente maior em partidas femininas $(7,1 \pm 2,0$ segundos) que em partidas masculinas $(5,2 \pm$ $1,8$ segundos $)^{(4,7)}$. O tipo de piso também influencia o tempo de troca de bolas. Em Roland Garros (quadra lenta de saibro), a duração de cada ponto é de aproximadamente $7,7 \pm 1,7$ segundos. Já, em Wimbledon (quadra rápida de grama), a duração média do ponto é de 4,3 $\pm 1,6$ segundos, pois os jogadores procuram com mais frequência o término do ponto próximo da rede ${ }^{(7)}$.

Além da análise do jogo, a fim de investigar a sobrecarga fisiológica imposta pela prática do tênis, alguns estudos utilizaram marcadores, como a concentração de lactato, o perfil da frequência cardíaca e o consumo máximo de oxigênio $\left(\mathrm{VO}_{2 \max }\right)$ durante as partidas ou em testes laboratoriais ${ }^{(8-14)}$.

Esses estudos revelaram que a sobrecarga fisiológica de uma partida é modesta. A intensidade média do esforço é, geralmente, menor que $70 \%$ do $\mathrm{VO}_{2 \max }{ }^{(10,11)}$ e inferior a $80 \%$ da frequência cardíaca máxima $a^{(12,13)}$. A concentração de lactato também permanece baixa (1,8-2,8 mmol...-1) durante a partida ${ }^{(12,13,14)}$. Provavelmente, os períodos de descanso possibilitam a rápida remoção do lactato, reduzindo a sua concentração plasmática.

Baseado nessas respostas, Bergeron et al. ${ }^{(15)}$ sugerem que o padrão de metabolismo observado na partida classificaria o tênis como uma atividade de longa duração de caráter aeróbio. Entretanto, Kovacs ${ }^{(3)}$ refuta a hipótese de que o tênis é uma atividade predominantemente aeróbia. Para esse autor, a resposta média dos indicadores fisiológicos $\left(\mathrm{VO}_{2 \text { max }}\right.$ lactato e $\left.\mathrm{FC}\right)$ observada durante a partida não retrata a natureza do tênis. Kovacs ${ }^{(3)}$ ressalta que a maior parte das ações específicas do tênis é de caráter explosivo, reforçando a hipótese de que esse esporte exige grande participação do sistema anaeróbio.

$\operatorname{Kovacs}^{(3)}$ ainda argumenta que a continua degradação de creatina fosfato exigirá maior participação do sistema glicolítico, resultando no aumento da concentração de lactato. De fato, a concentração de lactato pode aumentar, significativamente, para 8 a $11 \mathrm{mmol}^{-L^{-1}}$ durante pontos mais longos e disputados ${ }^{(16)}$. Essa resposta sugere uma contribuição significativa do metabolismo anaeróbio láctico em algumas situações ao longo da partida( ${ }^{(3)}$.
Apesar da controvérsia sobre a predominância dos sistemas energéticos no tênis, as informações disponíveis sugerem que durante as ações específicas é utilizada, predominantemente, a energia oriunda do metabolismo anaeróbio aláctico-láctico(10-14). Já nos períodos de intervalo, o sistema aeróbio, provavelmente, será priorizado, com intuito de promover a fosforilação da creatina fosfato e a remoção do lactato ${ }^{(3)}$.

O estudo das características específicas do tênis indica que todos os sistemas energéticos são utilizados durante uma partida. Esse padrão heterogêneo de ativação dos sistemas energéticos reforça a hipótese de que o CHO é um importante combustível para a prática do tênis. Baseado nessa suposição, recomenda-se elevada ingestão desse nutriente. Burke et al. ${ }^{(17)}$ recomendam a ingestão de cinco a sete gramas de $\mathrm{CHO}$ por quilograma por dia para atletas em fase de treinamento de intensidade média a elevada. Já no período de competição, segundo os mesmos autores, esse consumo deve aumentar para sete a 10 gramas de $\mathrm{CHO}$ por quilograma por dia, com a finalidade de manter o nível adequado dos estoques de glicogênio.

Durante os torneios/competições, os tenistas jogam em diferentes países, que muitas vezes apresentam padrões alimentares distintos do seu habitual, o que dificulta a manutenção de estratégias nutricionais eficazes $^{(18)}$. Essas alterações no padrão de alimentação, por exemplo, podem comprometer os estoques de glicogênio durante a fase competitiva e, consequentemente, afetar o desempenho(18). Além disso, as partidas durante os torneios são separadas por curtos intervalos de descanso ( 24h) que podem inviabilizar o completo restabelecimento dos estoques de glicogênio. Considerando a recomendação proposta por Burke et al.(17), a suplementação de CHO nesse período seria uma estratégia alternativa para atingir a elevada necessidade de CHO. Além disso, a partida de tênis possui períodos de descanso de forma estruturada que facilitariam o consumo desse nutriente ${ }^{(18,19)}$. Apesar do grande interesse relacionado com o desenvolvimento de estratégias ergogênicas, a investigação sobre o efeito da suplementação de CHO no tênis é limitada.

\section{Suplementação de carboidrato no exercício de endurance}

A relevância do $\mathrm{CHO}$ como substrato energético para o exercício de endurance já é conhecida desde o início do século 20. Considerando que os estoques endógenos de $\mathrm{CHO}$ são limitados e que a baixa disponibilidade desse nutriente afeta negativamente o desempenho

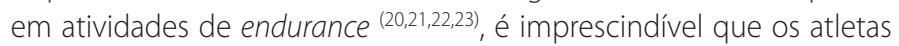
adotem estratégias adequadas para o consumo desse macronutriente antes, durante e depois dos treinos/competições. Krogh e Lindhardt ${ }^{(24)}$ foram os primeiros cientistas a reconhecer a importância do $\mathrm{CHO}$ como combustível para o exercício. Esses pesquisadores relataram que indivíduos submetidos a dieta rica em $\mathrm{CHO}$ apresentaram menor percepção subjetiva de esforço em comparação com a situação na qual os mesmos haviam consumido dieta rica em lipídios (24).

Em 1923, Levine et al. ${ }^{(25)}$ observaram redução da glicemia em corredores após a maratona de Boston. Para esses pesquisadores, a queda da glicemia era uma possível causa da fadiga. A fim de testar essa hipótese, no ano subsequente, corredores realizaram uma ingestão elevada de $\mathrm{CHO}$ antes e durante a corrida. Essa estratégia preveniu a hipoglicemia e aumentou, significativamente, o desempenho desses atletas ${ }^{(25)}$.

Posteriormente, na década de 30, Christensen ${ }^{(26)}$ mostrou que o aumento da intensidade do exercício era associada ao aumento na taxa de utilização do CHO endógeno. Esses achados foram corroborados, mais tarde em 1960, com a introdução da técnica de biopsia muscular(27,28). Esses estudos demonstraram, pela primeira vez, que o aumento dos estoques de glicogênio através de manipulações dietéticas estava relacionado com o aumento da capacidade de endurance. Desde então, os efeitos ergogênicos do CHO no exercício de endurance vêm sendo, amplamente, explorados por pesquisadores ${ }^{(22,23)}$. Jeukendrup ${ }^{(23)}$ relata 
que muitos estudos continuam trazendo evidências sobre o efeito ergogênico do consumo de CHO durante o exercício de endurance. Os estudos mais recentes verificaram efeitos positivos de suplementação de $\mathrm{CHO}$ em exercícios de alta intensidade $\left(75 \%\right.$ do $\mathrm{VO}_{2 \max }$ ) com duração de aproximadamente 60 minutos ${ }^{(22,23,29)}$. Jeukendrup et al. ${ }^{(29)}$ investigaram o efeito da ingestão de $\mathrm{CHO}$ e encontraram melhora do desempenho em atletas de ciclismo durante um teste com duração de 60 minutos.

Apesar de diversas pesquisas relatarem resultados positivos da suplementação de $\mathrm{CHO}^{(23,30,31)}$, Plamer et al. ${ }^{(32)}$ não verificaram benefício da suplementação da suplementação de $\mathrm{CHO}$ durante o exercício de curta duração ( 30 minutos). Em outro estudo recente, Jeukendrup et al. ${ }^{(33)}$ também não observaram aumento no desempenho de ciclistas durante o exercício realizado em alta intensidade (85-90\% $\mathrm{VO}_{2 \max }$ ) e duração de aproximadamente 30 minutos. Esses resultados reforçam a hipótese de que o efeito ergogênico do $\mathrm{CHO}$ é evidenciado em exercícios de endurance com duração superior a 60 minutos.

A controvérsia de resultados, provavelmente, está associada à dificuldade de reprodutibilidade dos protocolos utilizados ${ }^{(23,33)}$, uma vez que a forma de mensurar desempenho é complexa e suscetível a variáveis, tais como, a dieta, as condições climáticas, a capacidade física e a motivação dos indivíduos selecionados para participar do estudo. Alguns autores acreditam que a ausência do efeito ergogênico pode ser explicada pelo fato de o exercício de curta duração não depletar os estoques endógenos de $\mathrm{CHO}$ e/ou pelo fato de a quantidade de $\mathrm{CHO}$ ingerida ser insuficiente ${ }^{(33)}$.

Contudo, Jeukendrup ${ }^{(23)}$ reporta que a maioria dos estudos demonstra, de forma convincente, que a suplementação de CHO maximiza o desempenho de endurance. O mesmo ressalta que os possíveis mecanismos responsáveis pelo aumento da performance são: 1) manutenção da glicemia, 2) efeito poupador de glicogênio, 3) síntese de glicogênio em exercícios de baixa intensidade e 4) atraso/atenuação da fadiga central(23).

Além do aumento da performance, a suplementação de CHO também estaria relacionada com a atenuação da imunossupressão após exercícios de longa duração ${ }^{(34)}$. As investigações que avaliaram a supressão do sistema imunológico pós-exercício apontam a hipoglicemia como possível causa, uma vez que a redução da glicose circulante estimulará a secreção de cortisol. Esse hormônio reduz as concentrações de glutamina e imunoglobulina A (lgA), aumentando a suscetibilidade a infecção(35,36). Diversos autores sugerem que o consumo de $\mathrm{CHO}$ antes e durante o exercício prolongado é capaz de atenuar a liberação de cortisol e, consequentemente, a supressão da atividade do sistema imunológico(37-40).

\section{Suplementação de carboidrato no tênis}

Apesar do extenso número de evidências relacionadas com o efeito ergogênico do $\mathrm{CHO}$ sobre o desempenho de endurance, ainda não existe consenso de que essa estratégia maximize a performance no tênis. Os prováveis mecanismos que poderiam explicar o aumento do desempenho no tênis induzido pelo consumo de $\mathrm{CHO}$ são semelhantes aos propostos para o exercício de endurance.

Entretanto, no tênis, não existem estudos a respeito do efeito da suplementação de $\mathrm{CHO}$ sobre o metabolismo dos estoques de glicogênio. Também devido a limitações metodológicas, não existem estudos sobre o efeito da suplementação de $\mathrm{CHO}$ na etiologia da fadiga central nesse esporte. Logo, a maioria dos estudos que investigaram o efeito da suplementação de $\mathrm{CHO}$ no tênis avaliou, apenas, o comportamento da glicemia durante treinos/partidas. No entanto, os resultados desses estudos sobre a manutenção da glicemia durante a prática do tênis são controversos ${ }^{(15,41-43)}$. Bergeron et al. ${ }^{(15)}$ relataram que a concentração de glicose dos jogadores permaneceu estável durante uma partida com duração inferior a 90 minutos. Surpreendentemente, Mitchell et al. ${ }^{(41)}$ também não observaram redução da glicemia após uma partida com duração de 180 minutos.
Entretanto, Burke et al. ${ }^{(42)}$ verificaram redução da concentração plasmática de glicose durante uma simulação de jogo com duração de 120 minutos. Essa queda na concentração de glicose também foi observada em outros modelos de exercício com duração superior a 90 minutos ${ }^{(44,45)}$. Provavelmente, a redução da glicemia foi decorrente do aumento da demanda imposta pelo músculo esquelético e/ou menor liberação/produção hepática de glicose (via glicogenólise e gliconeogênese).

Os achados de Burke et al. ${ }^{(42)}$ tornam os resultados reportados por Mitchell et al. ${ }^{(41)}$ surpreendentes. Mitchell et al. ${ }^{(41)}$ acreditam que a partida de 180 minutos não provocou demanda metabólico-energética significativa que pudesse afetar a estabilidade da glicemia. Esses autores ainda sugerem que nas referidas condições (partida de tênis com duração de 180 minutos), o consumo adicional de $\mathrm{CHO}$ não seria necessário. Vale lembrar que no tênis o tempo real de jogo é de aproximadamente $20 \%$ do tempo total ${ }^{(3)}$. Portanto, partindo desse pressuposto, é provável que o tempo real de esforço no estudo de Mitchell et al. ${ }^{(41)}$ tenha sido inferior a 40 minutos.

Ainda sobre o controle da glicemia na prática do tênis, outro estudo interessante comparou as respostas referentes a essa variável durante o treino e a competição de tênis ${ }^{(43)}$. Ferrauti et al. ${ }^{(43)}$ demonstraram que as condições de competição elevam a concentração de glicose no sangue comparadas com as de treinamento. $\mathrm{O}$ aumento observado durante a competição pode ser explicado pelo maior tônus simpático e maior liberação de hormônios catabólicos (ex. adrenalina e glucagon) ${ }^{(46)}$, em resposta ao estresse competitivo ${ }^{(47)}$. O aumento na concentração plasmática destes hormônios, por sua vez, potencializaria a glicogenólise, promovendo aumento da glicemia. Mas, deve-se enfatizar que nenhuma conclusão definitiva pode ser tomada a partir desse único estudo, principalmente, devido à falta de padronização das condições de competição.

A literatura atual não apresenta consenso sobre o comportamento da glicemia em resposta à prática do tênis. Além disso, é importante ressaltar que a relação entre a queda da glicemia e a redução do desempenho também é controversa. Estudos iniciais sugeriram que a redução da glicemia estava relacionada com a etiologia da fadiga no exercício de endurance ${ }^{(25,26,45)}$. Entretanto, a investigação mais profunda sobre o assunto falhou em confirmar essa associação entre a glicemia e fadiga nesse tipo de atividade ${ }^{(48,49)}$. Mais especificamente no tênis, essa hipótese também precisa ser mais investigada.

Burke et al. ${ }^{(42)}$ verificaram que o consumo de $\mathrm{CHO}$ manteve a glicemia e promoveu aumento da qualidade dos golpes na partida de tênis com duração de 120 minutos. Em concordância aos achados de Burke et al. ${ }^{(42)}$, Vergauwen et al. ${ }^{(19)}$ concluíram que o consumo de $\mathrm{CHO}$ melhorou a precisão e diminuiu a taxa de erro durante os estágios finais do jogo de tênis. Contudo, não é possível afirmar que a manutenção da glicemia foi responsável pelo aumento do desempenho, uma vez que essa variável não foi avaliada no referido estudo(19).

Embora, o consumo de $\mathrm{CHO}$ promova aumento da concentração de glicose durante a partida de tênis, o desempenho dos jogadores nem sempre é potencializado ${ }^{(41,50)}$. Ferrauti et al. ${ }^{(50)}$ não observaram aumento da precisão de golpes após uma partida de 240 minutos no grupo suplementado com CHO em comparação com o grupo placebo. Nesse mesmo estudo, a suplementação de CHO também não afetou o número de games ganhos durante a partida. É importante mencionar que o grupo placebo apresentou discreto declínio na glicemia ao final dos 240 minutos, porém, o desempenho desses tenistas não foi prejudicado ${ }^{(50)}$. No estudo de Mitchell et al. ${ }^{(41)}$, a suplementação de CHO também não afetou o desempenho durante uma partida de 180 minutos. Conforme mencionado anteriormente, nesse estudo não foi detectada alteração significativa da glicemia após uma partida de 180 minutos no grupo placebo. Portanto, as evidências disponíveis sobre o efeito a suplementação de $\mathrm{CHO}$ no tênis são inconclusivas ${ }^{(19,41,42,43,50)}$. 
Outro possível benefício do consumo de $\mathrm{CHO}$ para tenistas pode estar relacionado com a funcionalidade do sistema imunológico. Conforme previamente observado por Novas et al.(51), o período de treinamento/competições de tênis promove a redução da concentração de $\lg A$ e o aumento da incidência de infecções no trato respiratório durante o período de 12 semanas(51).

A fim de investigar o possível efeito do consumo de $\mathrm{CHO}$ sobre a resposta imunológica, Nieman et al.(52) avaliaram a concentração de IgA em tenistas. Nesse estudo foi observado que a concentração de IgA permanecia inalterada, após duas sessões de treino de tênis com 120 minutos de duração. Entretanto, no referido estudo, não houve grupo placebo, todos os tenistas foram suplementados com $\mathrm{CHO}$ durante o experimento. Esse delineamento experimental compromete a conclusão sobre o efeito da suplementação de $\mathrm{CHO}$ na resposta imunológica de tenistas.

\section{CONCLUSÃO}

A literatura atual apresenta escasso número de estudos sobre o efeito da suplementação de $\mathrm{CHO}$ no desempenho de tenistas. Os resultados controversos, reportados em poucos estudos, não permitem que a pergunta levantada no título do artigo seja respondida ade-

\section{REFERÊNCIAS BIBLIOGRÁFICAS}

1. Fernandez J, Mendez-villanueva A, Pluim BM. Intensity of tennis match play. Br J Sports Med 2006;40:387-91. 2. ITF. Official rules of tennis. Chicago: Triumph Books; 2002.

3. Kovacs MS. Tennis physiology training the competitive athlete. Sports Med 2007;37:189-98.

4. Kovacs M. A comparision of work/rest intervals in men's professional tennis. Med Sci Tennis 2004;3:10-1.

5. Parsons LS, Jones MT. Development of speed, agility and quickness for tennis athletes. Strength Cond 1998;20:14-9

6. Ferrauti A, Weber K, Wright PR. Endurance: basic, semi-specific and specific. In: Reid M, Quinn A, Crespo M, editors. Strength and conditioning for tennis. London: ITF, 2003. p.93-111.

7. Donoghue PO, Ingram B. A notational analysis of elite tennis strategy. J Sports Sci 2001;19:107-15

8. Ferrauti A, Schulz H, Struder HK, Heck H, Weber K. Metabolism in tennis and running with similar oxygen uptake and duration. Int J Sports Med 1998;19.

9. Novas AMP, Rowbottom DG, Jenkins DG. A practical method of estimating energy expenditure during tennis play. J Sci Med Sport 2003;6:40-50.

10. Konig D, Hounker M, Schmid A, Halle M, Berg A, Keul J. Cardiovascular, metabolic, and hormonal parameters in professional tennis players. Med Sci Sports Exerc 2000;33:654-8.

11. Christmass MA, Richmond SE, Cable NT, Arthur PG, Hartmann PE. Exercise intensity and metabolic response in singles tennis. J Sports Sci 1998;16:739-47.

12. Davey PR, Thorpe RD, Williams C. Simulated tennis matchplay in a controlled environment. J Sports Sci 2003;21:459-67.

13. Mendez-Villanueva J, Fernandez-Fernandez A, Bishop D, Fernandez-Garcia B, Terrados N. Activity patterns, blood lactate concentrations and ratings of perceived exertion during a professional singles tennis tournament. Br J Sports Med 2007;41:296-300.

14. Fernandez J, Fernandez-Garcia B, Mendez-Villanueva A, Terrados N. Exercise intensity in tennis: simulated match play versus training drills. Med Sci Tennis 2005;10:6-7.

15. Bergeron MF, Maresh CM, Kraemer WJ. Tennis: a physiological profile during match play. Int J Sport Med 1991;12:474-9.

16. Smekal G, Von Duvillard SP, Rihacek C, Pokan R, Hofman P, Baron R, et al. A physiological profile of tennis match play. Med Sci Sports Exerc 2001;33:999-1005

17. Burke LM, Cox GR, Culmmings NK. Guidelines for daily carbohydrate intake: do athletes achieve them? Sports Med 2001;31:267-99.

18. Kovacs M. Carbohydrate intake and tennis: are there benefits? Br J Sports Med 2006;40:1-5.

19. Vergauwen L, Brouns F, Hespel P. Carbohydrate supplementation improves stroke performance in tennis. Med Sci Sports Exerc 1998:30:1289-95.

20. Coyle EF. Carbohydrate supplementation during exercise. J Nutr 1992;122:788-95.

21. Coyle EF. Substrate utilization during exercise in active people. Am J Clin Nutr 1995;61:9685-79S.

22. Jeukendrup AE. Modulation of carbohydrate and fat utilization by diet, exercise and environment. Biochem Soc Trans 2003;31:122-37.

23. Jeukendrup AE. Carbohydrate intake during exercise and performance. Nutr 2004;20:669-77.

24. Krogh A, Lindhard J. The relative value of fat and carbohydrate as sources of muscular energy. Biochem J 1920;14:290-6.

25. Levine SA, Gordon B, Derick CL. Some changes in chemical constituents of blood following a marathon race. JAMA 1924;82:1778-82

26. Christensen EH. Der Stoffwechsel und die Respiratorischen Funktionen bei schwerer körperlicher Arbeit Scand Arch Physiol 1932;81:160-5.

27. Bergstrom J, Hultman E. Muscle glycogen synthesis after exercise: an enhancing factor localized in muscle cells in man. Nature 1966;210:309-14. quadamente. Nos estudos de Burke ${ }^{(42)}$ e Vergauwen ${ }^{(19)}$ foi observado aumento na qualidade dos golpes, principalmente, ao final do experimento. Já nos estudos de Ferrauti et al. ${ }^{(50)}$ e Mitchell et al. ${ }^{(41)}$, o consumo de $\mathrm{CHO}$ não potencializou o desempenho de tenistas. A controvérsia observada nos estudos é, provavelmente, consequência de condições experimentais diferentes, tais como: a duração do treino/jogo/teste, os parâmetros utilizados para medir desempenho, o conteúdo inicial dos estoques de glicogênio e a análise/controle da dieta antes do experimento. Estudos adicionais, em condições similares às vivenciadas em torneios, precisam ser conduzidos a fim de avaliar o efeito da suplementação de $\mathrm{CHO}$ no tênis.

\section{AGRADECIMENTO}

Rodrigo Vitasovic Gomes agradece à Capes pela bolsa de mestrado. Também agradecemos o apoio financeiro do CNPq (Projeto 563967/2008-0) e o incentivo/suporte fornecido pelo Prof. Dr. Antonio Hebert Lancha Júnior.

Todos os autores declararam não haver qualquer potencial conflito de interesses referente a este artigo.
28. Bergstrom J, Hultman E. A study of glycogen metabolism during exercise in man. Scand J Clin Invest 1967;19:218-23.

29. Jeukendrup A, Brouns F, Wagenmakers AJ, Saris WH. Carbohydrate-electrolyte feedings improve $1 \mathrm{~h}$ time trial cycling performance. Int J Sports Med 1997;18:125-9.

30. Carter J, Jeukendrup AE, Mundel T, Jones DA. Carbohydrate supplementation improves moderate and high-intensity exercise in the heat. Pflugers Arch 2003;446:211-6.

31. El-Sayed MS, Balmer J, Rattu AJ. Carbohydrate ingestion improves endurance performance during a $1 \mathrm{~h}$ simulated time trial. J Sports Sci 1997;15:223-7

32. Plamer GS, Clancy MC, Hawley JA, Rodger IM, Burke LM, Noakes TD. Carbohydrate ingestion immediately before exercise does not improve $20 \mathrm{~km}$ time trial performance in well trained cyclists. Int J Sports Med 1998;19:415-8.

33. Jeukendrup AE, Hopkins S, Aragón-Vargas LF, Hulston C. No effect of carbohydrate feeding on $16 \mathrm{~km}$ cycling time trial performance. Eur J Appl Physiol 2008;104:831-7.

34. Braun WA, Serge P, Duvillard V. Influence of carbohydrate delivery on the immune response during exercise and recovery from exercise. Nutrition 2004;20:645-50.

35. Nieman DC. Is infection risk linked to exercise workload? Med Sci Sports Exerc 2000;32:S406-11.

36. Gleeson M. Can nutrition limit exercise-induced immunodepression? Nutr Rev 2006;64:119-31.

37. Utter AC, Kang J, Nieman D, Dumke CL. Carbohydrate attenuates perceived exertion during intermittent exercise and recovery. Med Sci Sports Exerc 2007;39:880-5.

38. Bacurau R, Bassit R, Sawada L, Navarro F, Martins E, Costa R, et al. Carbohydrate supplementation during intense exercise and the immune response of cyclists. Clin Nutr 2002;21:423-9.

39. Gleeson M, Nieman DC, Pedersen BK. Exercise, nutrition and immune function. J Sport Sci 2004;22:115-25.

40. Davison G, Gleeson M. Influence of acute vitamin C and/or carbohydrate ingestion on hormonal, cytokine, and immune responses to prolonged exercise. Int J Sport Nutr Exerc Metab 2005;15:465-79.

41. Mitchell JB, Cole JK, Grandjean PW, Sobczak RJ. The effect of a carbohydrate beverage on tennis performance and fluid balance during prolonged tennis play. J Appl Sport Sci Res 1992;6:96-102.

42. Burke ER, Ekblom B. Influence of fluid ingestion and dehydration on precision and endurance performance in tennis. Athletic Trainer 1982;17:275-7.

43. Ferrauti A, Pluim BM, Busch T. Blood glucose responses and incidence of hypoglycaemia in elite tennis under practice and tournament conditions. J Sci Med Sport 2003;6:28-39.

44. Davis SN, Galasetti P, Wasserman DH. Effects of antecedent of hypoglycaemia on subsequent counterregulatory responses to exercise. Diabetes 2000;49:73-81.

45. Coggan AR, Coyle EF. Carbohydrate ingestion during prolonged exercise: effects on metabolism and performance. Exerc Sport Sci Rev 1991;19:1-40.

46. Kjaer M, Secher NH, Bach FW, Galbo H. Role of motor center activity for hormonal changes and substrate mobilization in humans. Am J Physiol 1987;253:R687-95.

47. Therminarias A, Dansou P, Chirpaz-Oddou MF, Gharib C, Quirion A. Hormonal and metabolic changes during a strenuous tennis match. Effect of ageing. Int J Sports Med 1991;12:10-6.

48. Ivy JL, Costill DL, Fink WJ, Lower RW. Influence of caffeine and carbohydrate feedings on endurance performance. Med Sci Sports 1979;11:6.

49. Felig $\mathrm{P}$, Cherif A, Minagawa A, Wahren J. Hypoglycemia during prolonged exercise in normal men. N Engl J Med 1982;306:895-9.

50. Ferrauti A, Weber K, Strüder HK. Metabolic and ergogenic effects of carbohydrate and caffeine beverages in tennis. J Sports Med Phys Fitness 1997;37:258-66.

51. Novas AMP, Rowbotton DG, Jenkins DG. Tennis, incidence of URTI and salivary IgA. Int J Sports Med 2003;24:223-9.

52. Nieman DC, Kernodle MK, Henson DA, Sonnenfeld G, Morton DS. The acute response of the immune system to tennis drills in adolescents athletes. Res Q Exerc Sport 2000:71:403-8. 